\title{
Globale Integration lokal integrierbarer Vektorfelder
}

\begin{abstract}
Serge Lang
Serge Lang wurde 1927 in Paris geboren, wo er auch seine ersten Schuljahre absolvierte. Die weitere Ausbildung erhielt er dann allerdings in den Vereinigten Staaten, wo er das California Institute of Technology (Caltech) und die University of Princeton besuchte. Hier erhielt er das Doktorat in Mathematik im Jahre 1951. Nach Aufenthalten am Institute for Advanced Study in Princeton und an der University of Chicago war er von 1955 bis 1970 Professor an der Columbia University in New York. Gastprofessuren in Princeton und Harvard folgten, und 1972 wurde er Professor an der Yale University. Seine Interessen sind weitgespannt, aber sein Hauptinteresse gehörte immer der Mathematik, besonders der Zahlentheorie. Bis anhin hat er 34 Bücher und über 70 Forschungsartikel veröffentlicht.
\end{abstract}

Wir betrachten in der Ebene $\mathbf{R}^{2}$ ein Gebiet (zusammenhängende offene Menge) $U$ und in $U$ ein Vektorfeld

$$
F: U \rightarrow \mathbf{R}^{2}, \quad F(x, y)=(p(x, y), q(x, y)) .
$$

Wir setzen dabei voraus, dass die Komponentenfunktionen $p$ und $q$ stetig differenzierbar sind. Ferner sei in $U$ ein stückweise stetig differenzierbarer Weg $\gamma:[a, b] \rightarrow U$ gegeben mit Anfangspunkt $P=\gamma(a)$ und Endpunkt $Q=\gamma(b)$. Unter diesen Voraussetzungen ist

Der Cauchysche Integralsatz der klassischen Funktionentheorie ist, zusammen mit seiner Verallgemeinerung, dem Residuensatz, eines jener mathematischen Resultate, die immer wieder im Zentrum neuer Überlegungen stehen. Überraschende Verbindungen zeigen sich zu verschiedenen anderen Gebieten, zum Beispiel zur reellen Analysis und zur algebraischen Topologie. Nur schon das Offenlegen von derartigen Zusammenhängen führt $\mathrm{zu}$ einer tieferen Einsicht, und neue Beweise und neue Resultate sind die Folge. Serge Lang geht in seinem Beitrag einigen dieser Beziehungen nach. Der Problemkreis wird, auf das Wesentlichste reduziert, in einen weiten Zusammenhang eingeordnet. Nicht nur gewinnt der Gegenstand auf diese Weise an Transparenz, sondern es zeigen sich auch neue allgemeine Resultate, die den Cauchyschen Integralsatz und den Residuensatz als einfache Folgerungen beinhalten. - Der Beitrag basiert auf einem Vortrag von Serge Lang an der ETH Zürich, den er am 1. Juni 1995 vor einer Zuhörerschaft von jungen Studierenden gehalten hat. ust 
das Integral $\int_{\gamma} F$ definiert (siehe Figur 1). Bekanntlich hängt im allgemeinen der Wert dieses Integrals vom Weg $\gamma$ - und nicht nur von Anfangs- und Endpunkt des Weges ab.

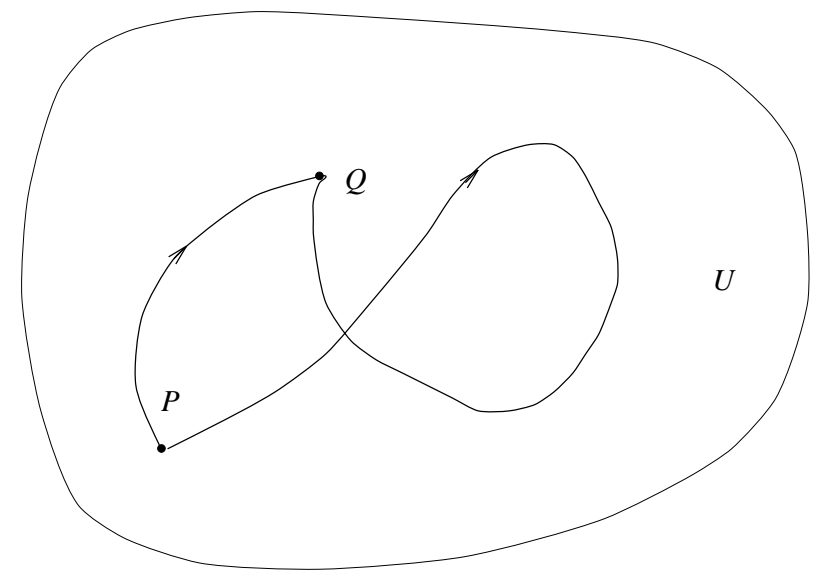

Fig. 1

Für eine wichtige Klasse von Vektorfeldern ist die Situation allerdings einfacher. Eine Funktion $\phi: U \rightarrow \mathbf{R}$ heisst ein Potential des Vektorfeldes $F$, wenn $F$ der Gradient von $\phi$ ist:

$$
(p, q)=\left(\frac{\partial \phi}{\partial x}, \frac{\partial \phi}{\partial y}\right)
$$

Ein Vektorfeld, zu dem ein Potential existiert, heisst ein Potentialfeld. Ist $F$ ein Potentialfeld mit Potential $\phi$, so folgt - wie eine einfache Rechnung zeigt -

$$
\int_{\gamma} F=\phi(Q)-\phi(P)
$$

Für Potentialfelder hängt der Wert des Integrals $\int_{\gamma} F$ folglich nur von Anfangs- und Endpunkt des Weges $\gamma$ ab. Es zeigt sich, dass die Eigenschaft (1) die Potentialfelder auch charakterisiert. Es gilt nämlich das folgende grundlegende Resultat:

Satz 1 Das Vektorfeld $F$ besitzt genau dann ein Potential auf $U$, wenn für Punkte $P$ und $Q$ in $U$ und für in $U$ verlaufende Wege $\gamma$ mit Anfangspunkt $P$ und Endpunkt $Q$ das Integral $\int_{\gamma} F$ von $\gamma$ unabhängig ist.

Wir führen hier den Beweis dieses in der Vektoranalysis wohlbekannten Satzes nicht vollständig durch, sondern begnügen uns damit, die wesentlichsten Schritte in Erinnerung zu rufen. Um das Potential $\phi$ zu definieren, wählen wir in $U$ einen Punkt $P_{0}$. Ist $Q$ ein Punkt in $U$ und $\gamma$ ein Weg mit Anfangspunkt $P_{0}$ und Endpunkt $Q$, so setzt man

$$
\phi(Q)=\int_{\gamma} F .
$$


Wegen der Unabhängigkeit von $\gamma$ definiert dies in der Tat eine Funktion $\phi$ auf $U$, die wie man unschwer nachweist - ein Potential des Vektorfeldes $F$ ist. Für die Einzelheiten des Beweises konsultiere man z.B. [L1], Chapter 15, Theorem 4.2.

Satz 1 lässt sich auch etwas anders ausdrücken. Ein Weg $\gamma$ heisst geschlossen, wenn sein Endpunkt mit seinem Anfangspunkt übereinstimmt.

Satz $1^{\prime}$ Das Vektorfeld $F$ besitzt genau dann ein Potential auf $U$, wenn für alle geschlossenen Wege $\gamma$ in $U$ gilt: $\int_{\gamma} F=0$.

Um die Äquivalenz der beiden Aussagen einzusehen, muss man nur beachten, dass aus zwei Wegen von $P$ nach $Q$ ein geschlossener Weg gebildet werden kann.

Aus einem Satz der elementaren Analysis (Satz von Schwarz) folgt, dass für die Komponenten $(p, q)$ eines Potentialfeldes $F$ mit Potential $\phi$,

$$
F=(p, q)=\left(\frac{\partial \phi}{\partial x}, \frac{\partial \phi}{\partial y}\right)
$$

die Gleichung

$$
\frac{\partial p}{\partial y}=\frac{\partial q}{\partial x}
$$

erfüllt ist, weil

$$
\frac{\partial p}{\partial y}=\frac{\partial^{2} \phi}{\partial x \partial y}=\frac{\partial^{2} \phi}{\partial y \partial x}=\frac{\partial q}{\partial x}
$$

gelten muss. Die Bedingung (2) ist also eine notwendige Bedingung dafür, dass das Vektorfeld $F=(p, q)$ ein Potential $\phi$ besitzt; man spricht deshalb auch etwa von der Integrabilitätsbedingung. In der elementaren Analysis beweist man ohne grosse Mühe, dass unter bestimmten zusätzlichen Bedingungen, welche das Gebiet $U$ betreffen, die Integrabilitätsbedingung für die Existenz eines Potentials auch hinreichend ist. Es gilt:

Satz 2 Es sei $F$ ein Vektorfeld auf $U$, welches die Integrabilitätsbedingung (2) erfüllt, und es sei $R$ ein ganz in $U$ liegendes Rechteck, bzw. D eine ganz in $U$ liegende Kreisscheibe. Dann besitzt F im Rechteck R bzw. in der Kreisscheibe D ein Potential $\phi$.

Beweis. Wir fixieren einen (beliebigen) Punkt $\left(x_{0}, y_{0}\right)$ des Rechtecks $R$ bzw. den Mittelpunkt $\left(x_{0}, y_{0}\right)$ der Kreisscheibe $D$ und definieren für $(x, y)$ in $R$ bzw. $D$

$$
\phi(x, y)=\int_{x_{0}}^{x} p(t, y) d t+\int_{y_{0}}^{y} q\left(x_{0}, u\right) d u .
$$

Das zweite Integral ist nicht von $x$ abhängig. Nach dem Fundamentalsatz der Infinitesimalrechnung erhält man dann für die Ableitung von $\phi$ nach $x$

$$
\frac{\partial}{\partial x} \phi(x, y)=p(x, y)
$$


Um die Ableitung von $\phi$ nach $y$ zu bestimmen, darf man unter den gegebenen Voraussetzungen unter dem Integral ableiten. Damit ergibt sich

$$
\begin{aligned}
\frac{\partial}{\partial y} \phi(x, y) & =\int_{x_{0}}^{x} \frac{\partial}{\partial y} p(t, y) d t+q\left(x_{0}, y\right) \\
& =\int_{x_{0}}^{x} \frac{\partial}{\partial t} q(t, y) d t+q\left(x_{0}, y\right) \\
& =q(x, y)-q\left(x_{0}, y\right)+q\left(x_{0}, y\right) \\
& =q(x, y) .
\end{aligned}
$$

Es ist also $\phi$ ein Potential von $F$.

Ein Vektorfeld, das die Integrabilitätsbedingungen (2) erfüllt, heisst lokal integrierbar. Man könnte diese Definition sogar etwas abschwächen, indem man verlangt, dass jeder Punkt $P$ eine Umgebung besitzt, in der $F$ ein Potential hat. Wir bleiben aber hier aus Gründen der Einfachheit bei der oben angegebenen Definition.

Im allgemeinen Fall ist die Integrabilitätsbedingung für die Existenz eines Potentials nicht hinreichend. Ein explizites Beispiel ist durch das Vektorfeld

$$
G(x, y)=\left(\frac{-y}{x^{2}+y^{2}}, \frac{x}{x^{2}+y^{2}}\right)=\left(\frac{-y}{r^{2}}, \frac{x}{r^{2}}\right), \quad \text { wo } \quad r^{2}=x^{2}+y^{2},
$$

gegeben, das auf der punktierten Ebene $\mathbf{R}^{2} \backslash\{O\}$ definiert ist. Wie man leicht nachrechnet, erfüllt das Vektorfeld $G$ die Integrabilitätsbedingung (2), es ist also lokal integrierbar. Andererseits kann $G$, wie wir jetzt nachweisen, global kein Potential besitzen. Wir werden nämlich zeigen, dass es geschlossene Wege $\gamma$ gibt, für die das Integral $\int_{\gamma} G$ nicht Null ist. Dazu berechnen wir zuerst $p d x+q d y$ in Polarkoordinaten $r, \theta$. Es ergibt sich

$$
p d x+q d y=d \theta,
$$

denn mit $x=r \cos \theta, y=r \sin \theta$ erhält man

$$
p d x+q d y=-\frac{r \sin \theta}{r^{2}}(\cos \theta d r-r \sin \theta d \theta)+\frac{r \cos \theta}{r^{2}}(\sin \theta d r+r \cos \theta d \theta),
$$

und die rechte Seite reduziert sich sofort auf $d \theta$.

Es folgt aus dieser Rechnung, dass der Polarwinkel $\theta$ für $G$ lokal ein Potential ist: Für jedes ganz in $\mathbf{R}^{2} \backslash\{O\}$ liegende Rechteck $R$, bzw. für jede ganz in $\mathbf{R}^{2} \backslash\{O\}$ liegende Kreisscheibe $D$ ist $\theta$ ein Potential für $G$.

Ist $\gamma$ irgendein Weg, der $O$ nicht enthält, so liefert $\int_{\gamma} G$ das Integral über $d \theta$. Für einen geschlossenen Weg $\gamma$ in der punktierten Ebene $\mathbf{R}^{2} \backslash\{O\}$ ändert sich $\theta$ um ein ganzzahliges Vielfaches von $2 \pi$. Man erhält folglich

$$
\int_{\gamma} G=2 \pi k
$$

wo $k$ eine ganze Zahl ist. Insbesondere ist für den einmal im Gegenuhrzeigersinn durchlaufenen Kreis mit Mittelpunkt $O$ der Wert des Integrals $2 \pi$ und nicht Null. Wir nehmen (3) zum Anlass für die folgende Definition: 
Definition Es sei $\gamma$ ein geschlossener Weg in der Ebene, der $O$ nicht enthält. Dann definieren wir die Umlaufzahl $W(\gamma, O)$ von $\gamma$ bezüglich $O$ durch

$$
W(\gamma, O)=\frac{1}{2 \pi} \int_{\gamma} G
$$

Für einen beliebigen Punkt $P \in \mathbf{R}^{2}, P=\left(x_{0}, y_{0}\right)$ setzen wir

$$
G_{P}(x, y)=G\left(x-x_{0}, y-y_{0}\right)
$$

Für einen geschlossenen Weg $\gamma$ mit $P \notin \gamma$ ist die Umlaufzahl $W(\gamma, P)$ von $\gamma$ bezüglich $P$ durch

$$
W(\gamma, P)=\frac{1}{2 \pi} \int_{\gamma} G_{P}
$$

definiert.

Es wird sich in der Folge zeigen, dass das Vektorfeld $G$ und seine verschobenen Kopien $G_{P}$ das einzige Hindernis dafür bilden, dass Vektorfelder, welche die Integrabilitätsbedingung (2) erfüllen, in $U$ auch global ein Potential besitzen. Dieses überraschende Resultat basiert auf dem folgenden Satz 3. Wie eine tiefere Analyse, auf die wir hier nicht eingehen können, zeigt, handelt es sich dabei eigentlich um ein rein topologisches Resultat; es wird hier aber in einer analytischen Form ausgesprochen.

Satz 3 Es sei $\gamma$ ein geschlossener Weg in $U$, so dass für alle $P \notin U$ die Umlaufzahl $W(\gamma, P)$ Null ist. Es sei $F$ ein lokal integrierbares Vektorfeld in $U$. Dann gilt

$$
\int_{\gamma} F=0
$$

Wir werden weiter unten auf einige Anwendungen des Satzes 3 zurückkommen.

Der Beweis verläuft in mehreren Schritten. Der erste Schritt besteht in einer Reduktion auf "Rechteckswege". Wir nennen einen Weg $\gamma$ einen Rechtecksweg, wenn er sich aus endlich vielen Geradenstücken zusammensetzt, die parallel zu den Koordinatenachsen verlaufen. Wir behaupten:

Lemma 4 Gilt die Aussage von Satz 3 für Rechteckswege $\eta$, so gilt sie auch für beliebige (stückweise stetig differenzierbare) Wege $\gamma$.

Beweis. Wir überdecken den Weg $\gamma$ durch ganz in $U$ liegende offene Kreisscheiben $D_{i}, i=1,2, \ldots, N$, und wählen Kurvenpunkte $P_{i}$ mit $P_{i} \in D_{i} \cap D_{i-1}$, und ferner $P_{N+1}=P_{0} \in D_{N} \cap D_{1}$ (man beachte, dass der Weg $\gamma$ geschlossen ist). Das Wegstück $\gamma_{i}$ zwischen $P_{i}$ und $P_{i+1}$ ersetzen wir dann durch ein Wegstück $\eta_{i}$, das aus zwei geradlinigen Stücken parallel zu den beiden Koordinatenachsen besteht und ganz im Innern von $D_{i}$ 


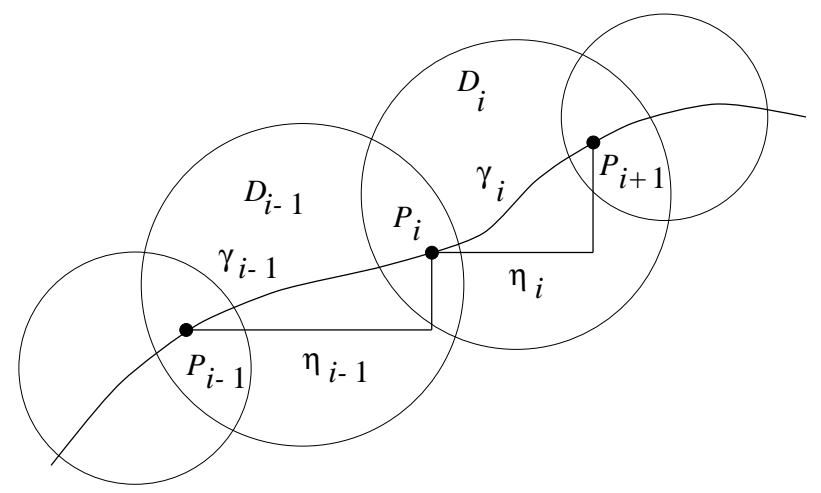

Fig. 2

verläuft (siehe Figur 2). Da das Vektorfeld $F$ lokal integrierbar ist, folgt

$$
\int_{\gamma_{i}} F=\int_{\eta_{i}} F, \text { für } i=1,2, \ldots, N
$$

und damit

$$
\int_{\gamma} F=\int_{\eta} F
$$

Analoges gilt für die (ebenfalls lokal integrierbaren) Vektorfelder $G_{P}$, die zur Berechnung der Umlaufzahl herangezogen werden. Daraus folgt für $P \notin U$

$$
W(\eta, P)=W(\gamma, P)=0 \text {. }
$$

Da dies für jedes $P \notin U$ zutrifft, folgt

$$
\int_{\eta} F=0
$$

falls die Aussage des Satzes 3 für Rechteckswege als richtig angenommen wird. Mit (4) ergibt sich somit

$$
\int_{\gamma} F=0
$$

wie in Lemma 4 behauptet.

In einem zweiten Schritt beweisen wir die Aussage des Satzes 3 für Rechteckswege. Es sei zuerst $R$ ein beliebiges, ganz in $U$ liegendes Rechteck, und $\partial R$ sei der im positiven Umlaufsinn durchlaufene Rand von $R$. Da das Vektorfeld $F$ lokal integrierbar ist, folgt aus unserem Satz $2 \int_{\partial R} F=0$.

Wenn der Weg $\gamma$ als Summe $\sum m_{i} \partial R_{i}$ gegeben ist, wobei für alle $i$ das Rechteck $R_{i}$ ganz in $U$ enthalten sei, so folgt

$$
\int_{\gamma} F=\sum_{i} m_{i} \int_{\partial R_{i}} F=0 .
$$

Das fundamentale Resultat ist nun das folgende; es beweist gemäss den obigen Bemerkungen die Aussage des Satzes 3 für Rechteckswege. 
Satz 5 Es sei $\gamma$ ein geschlossener Rechtecksweg in $U$. Es gelte $W(\gamma, P)=0$ für alle Punkte $P$ mit $P \notin U$. Dann existieren ganz in $U$ enthaltene Rechtecke $R_{i}$ und ganze Zahlen $m_{i} \in \mathbf{Z}$ mit

$$
\gamma=\sum_{i} m_{i} \partial R_{i} .
$$

Beweis. Ausgehend vom Rechtecksweg $\gamma$ zeichnen wir als erstes alle achsenparallelen Geraden, welche Geradenstücke des Weges $\gamma$ enthalten. Dies liefert eine Zerlegung der Ebene $\mathbf{R}^{2}$ in endlich viele endliche und unendliche Rechtecke. Wir zeichnen ferner vier weitere achsenparallele Geraden ein, die dazu dienen, die bei der so konstruierten Unterteilung entstandenen unendlichen Rechtecke abzuschneiden. Damit verläuft die gegebene Rechteckskurve so, dass in jeden Teilstück sowohl links wie rechts des Weges ein endliches Rechteck unserer Unterteilung liegt (siehe Figur 3). Es seien $R_{1}, R_{2}, \ldots, R_{N}$ die endlichen Rechtecke der so entstandenen Unterteilung. Wir wählen in jedem $R_{i}$ einen Punkt $P_{i}$ und definieren $m_{i}=W\left(\gamma, P_{i}\right)$. Dann behaupten wir

(a) Es gilt $\gamma=\sum_{i} m_{i} \partial R_{i}$.

(b) Für $m_{i} \neq 0$ ist $R_{i} \subseteq U$.

Diese beiden Behauptungen zusammen beweisen Satz 5.

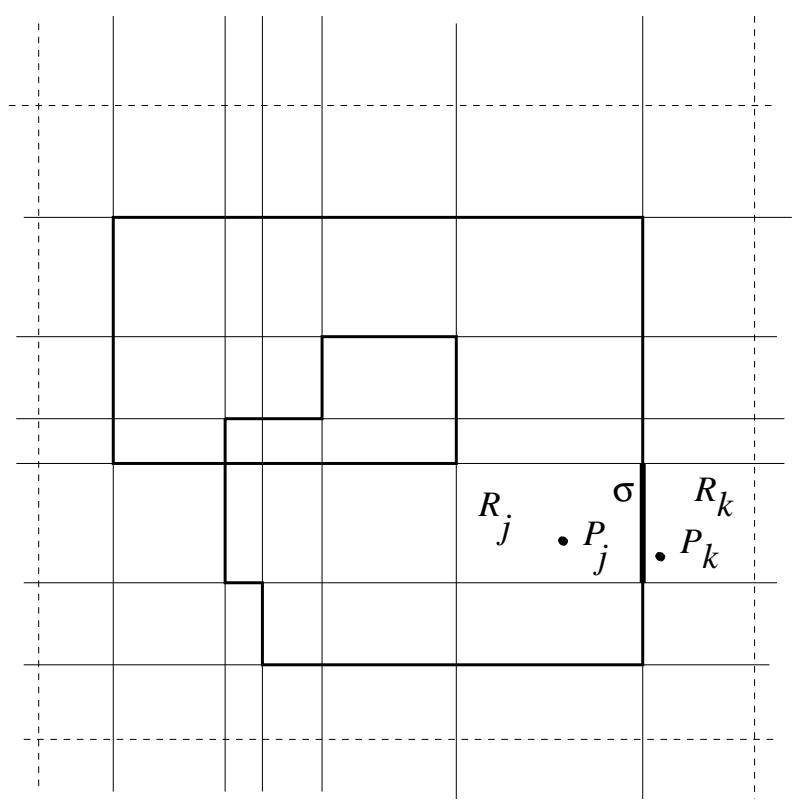

Fig. 3

Wir wenden uns zuerst der Behauptung (b) zu. Es sei $m_{i} \neq 0$. Laut Definition von $m_{i}$ ist die Umlaufzahl $W\left(\gamma, P_{i}\right)$ ungleich Null. Nun ist aber die Umlaufzahl einerseits stetig vom Punkt $P_{i}$ abhängig, andererseits eine ganze Zahl. Es ist also die Umlaufzahl auf 
zusammenhängenden Mengen konstant. Für $P$ im Innern des Rechtecks $R_{i}$ ist folglich $W(P, \gamma)=W\left(P_{i}, \gamma\right) \neq 0$. Daraus folgt $P \in U$, denn für $P \notin U$ gilt $W(P, \gamma)=0$ nach Voraussetzung. Für $P \in \partial R_{i}$ gilt dasselbe Argument, ausser im Fall $P \in \gamma$. Da aber $\gamma$ in $U$ liegt, folgt auch in diesem Fall $P \in U$. Es ergibt sich aus diesen Überlegungen, dass das ganze (abgeschlossene) Rechteck $R_{i}$ in $U$ liegt.

Um die Behaupung (a) zu beweisen, betrachten wir den Weg $\gamma^{\prime}$ mit

$$
\gamma^{\prime}=\gamma-\sum_{i} m_{i} \partial R_{i}
$$

Es ist zu zeigen, dass $\gamma^{\prime}$ kein gerades Teilstück von $\gamma$ mehr enthält.

Es sei $\sigma$ eine Seite des beliebigen Rechtecks $R_{k}$. Dann können wir schreiben

$$
\gamma^{\prime}=\gamma-\sum_{i} m_{i} \partial R_{i}=m \sigma+\text { Terme ohne } \sigma,
$$

wobei $m$ eine gewisse ganze Zahl ist. Betrachten wir den Weg $\gamma-\sum_{i} m_{i} \partial R_{i}-m \partial R_{k}$, so erhalten wir für die Umlaufzahl bezüglich $P_{k}$

$$
\begin{aligned}
W\left(\gamma-\sum_{i} m_{i} \partial R_{i}-m \partial R_{k}, P_{k}\right) & =W\left(\gamma, P_{k}\right)-\sum_{i} m_{i} W\left(\partial R_{i}, P_{k}\right)-m W\left(\partial R_{k}, P_{k}\right) \\
& =m_{k}-m_{k}-m \\
& =-m
\end{aligned}
$$

Im Weg $\gamma^{\prime \prime}=\gamma-\sum m_{i} \partial R_{i}-m \partial R_{k}$ kommt aber das Geradenstück $\sigma$ nicht mehr vor. In unserer Unterteilung gehörte $\sigma$ zum Rand von zwei Rechtecken: Liegt $R_{k}$ auf der einen Seite, so liege $R_{j}$ auf der anderen Seite von $\sigma$ (siehe Figur 3). Die Punkte $P_{k}$ und $P_{j}$ liegen dann für $\gamma^{\prime \prime}$ in einem zusammenhängenden Gebiet, so dass folgt

$$
\begin{aligned}
-m & =W\left(\gamma-\sum m_{i} \partial R_{i}-m \partial R_{k}, P_{k}\right) \\
& =W\left(\gamma-\sum m_{i} \partial R_{i}-m \partial R_{k}, P_{j}\right) \\
& =W\left(\gamma, P_{j}\right)-\sum_{i} m_{i} W\left(\partial R_{i}, P_{j}\right)-m W\left(\partial R_{k}, P_{j}\right) \\
& =m_{j}-m_{j}-0 \\
& =0 .
\end{aligned}
$$

Dies beweist unsere Behauptung (a).

Unser Beweis des Satzes 5 ist einem Beweis von Emil Artin für den Satz von Cauchy der (komplexen) Funktionentheorie nachgebildet (siehe [A]). Wenn man das obige Resultat im Kontext der rellen Analysis kennt, so sieht man sofort, wie es sich in den komplexen Fall übersetzen lässt. Die obigen Argumente liefern in der Tat den Hauptteil des globalen Satzes von Cauchy (siehe z.B. [L2], Chapter IV, Theorem 2.2). Dazu werden wir weiter unten noch einige Bemerkungen machen. Zuerst wenden wir uns aber einer anderen Anwendung zu (siehe Figur 4). 


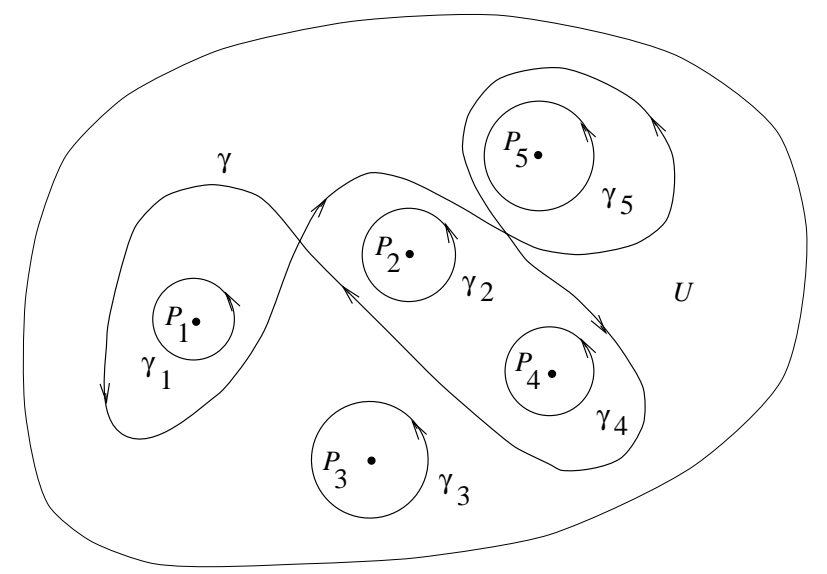

Fig. 4

Satz 6 Im Gebiet $U$ der Ebene sei ein geschlossener Weg $\gamma$ gegeben, welcher die Eigenschaft hat, dass für jeden ausserhalb von $U$ liegenden Punkt $P$ die Umlaufzahl $W(\gamma, P)$ Null ist. Es seien ferner $P_{1}, P_{2}, \ldots, P_{n}$ Punkte in $U$, die nicht auf $\gamma$ liegen. Für jedes $i=1,2, \ldots, n$ wählen wir eine ganz in $U$ enthaltene (kleine) Kreisscheibe $D_{i}$ mit Mittelpunkt $P_{i}$, und zwar so, dass keiner der Punkte $P_{k}$ mit $k \neq i$ in $D_{i}$ liegt. Die Randkurve $\gamma_{i}$ von $D_{i}$ orientieren wir im Gegenuhrzeigersinn. Wir setzen $m_{i}=W\left(\gamma, P_{i}\right)$. Es sei $U^{*}=U \backslash\left\{P_{1}, P_{2}, \ldots, P_{n}\right\}$, und $F$ sei ein in $U^{*}$ lokal integrierbares Vektorfeld. Dann gilt

$$
\int_{\gamma} F=\sum_{i=1}^{n} m_{i} \int_{\gamma_{i}} F
$$

Beweis. Wir setzen $C=\gamma-\sum m_{i} \gamma_{i}$. Ist $P$ ein Punkt ausserhalb $U$, so folgt nach obigem

$$
W(C, P)=W(\gamma, P)-\sum m_{i} W\left(\gamma_{i}, P\right)=0
$$

Für den Punkt $P_{k}$ an Stelle des Punktes $P$ erhält man $W\left(\gamma_{i}, P_{k}\right)=1$ für $i=k$ und $W\left(\gamma_{i}, P_{k}\right)=0$ für $i \neq k$. Damit folgt

$$
W\left(C, P_{k}\right)=W\left(\gamma, P_{k}\right)-m_{k}=0
$$

Auf das Gebiet $U^{*}$ und die Kurve $C$ wollen wir jetzt unseren Satz 3 anwenden. Dabei ist zu bemerken, dass die Aussage des Satzes 3 nicht nur für geschlossene Kurven gilt, sondern auch für Kurven, die - wie $C$ - endliche Summen von geschlossenen Kurven sind. Es folgt dann

$$
0=\int_{C} F=\int_{\gamma} F-\sum_{i}^{n} m_{i} \int_{\gamma_{i}} F,
$$

woraus sofort die Aussage des Satzes 6 folgt. 
Wir können - ähnlich wie in der (komplexen) Funktionentheorie - das Residuum eines lokal integrierbaren Vektorfeldes $F$ in einem Punkt $P_{i}$ definieren durch

$$
\operatorname{res}_{P_{i}}(F)=\frac{1}{2 \pi} \int_{\gamma_{i}} F
$$

wo $\gamma_{i}$ wie oben einen kleinen, im Uhrzeigersinn orientierten Kreis um $P_{i}$ bezeichnet. Das Residuum ist unter den Voraussetzungen des Satzes 6 unabhängig von $\gamma_{i}$. Mit dieser Definition lautet die Aussage von Satz 6

$$
\int_{\gamma} F=\sum 2 \pi m_{i} \cdot \operatorname{res}_{P_{i}}(F)
$$

Diese Terminologie wird in der Funktionentheorie bei der Darstellung des entsprechenden Resultates benützt, das vom Wegintegral einer meromorphen Funktion längs eines geschlossenen Weges in der komplexen Ebene handelt. Der Zusammenhang ist leicht beschrieben: Es seien $f$ eine komplexe Funktion und $\gamma$ ein Weg in der komplexen Ebene. Fasst man $\gamma$ als Weg in der reellen Ebene auf, so lassen sich Real- und Imaginärteil des komplexen Wegintegrals $\int_{\gamma} f(z) d z$ als Integral von zwei zweidimensionalen rellen Vektorfeldern längs $\gamma$ auffassen. Diese beiden Vektorfelder sind ausserdem lokal integrierbar, wenn $f$ die Cauchy-Riemannschen Differentialgleichungen erfüllt. Unser Satz 6 enthält also als Korollar das entsprechende Resultat der Funktionentheorie, den sogenannten Residuensatz (siehe z.B. [L2], Chapter VI, Theorem 1.2).

Eine weitere Anwendung betrifft die folgende Situation, die ebenfalls in der Funktionentheorie betrachtet wird. Wir formulieren allerdings nur das reelle Resultat, die Übersetzung ins Komplexe ist einfach. Für diesen Zweck nennen wir ein Gebiet $U$ einfach zusammenhängend, wenn für jeden Weg $\gamma$ und jeden Punkt ausserhalb $U$ die Umlaufzahl $W(\gamma, P)$ verschwindet, $W(\gamma, P)=0$. Es ist nicht schwer nachzuweisen, dass dies aus den sonst üblichen Definitionen von einfach zusammenhängend folgt.

Satz 7 Es sei $U$ ein einfach zusammenhängendes Gebiet der Ebene. Es seien $P_{1}, P_{2}, \ldots, P_{n}$ paarweise verschiedene Punkte in $U$, und es sei $U^{*}=U \backslash\left\{P_{1}, P_{2}, \ldots, P_{n}\right\}$. Wie oben setzen wir

$$
G(x, y)=\left(\frac{-y}{x^{2}+y^{2}}, \frac{x}{x^{2}+y^{2}}\right)
$$

und bezeichnen mit $G_{P_{i}}$ die Translation von $G$ nach $P_{i}$. Ist $F$ ein lokal integrierbares Vektorfeld auf $U^{*}$, so existieren Konstanten $a_{1}, a_{2}, \ldots, a_{n}$ und eine Funktion $\phi$ auf $U^{*}$, so dass

$$
F-\sum_{i=1}^{n} a_{i} G_{P_{i}}=\operatorname{grad} \phi .
$$


Beweis. Es sei $\gamma_{i}$ die im Uhrzeigersinn orientierte Peripherie eines kleinen Kreises mit Mittelpunkt $P_{i}$, der keinen weiteren der Punkte $P_{1}, P_{2}, \ldots, P_{n}$ enthält. Dann gilt

$$
\int_{\gamma_{i}} G_{P_{j}}= \begin{cases}2 \pi & \text { falls } i=j \\ 0 & \text { falls } i \neq j\end{cases}
$$

Wir setzen

$$
a_{i}=\frac{1}{2 \pi} \int_{\gamma_{i}} F \quad \text { und } \quad F_{1}=F-\sum_{i=1}^{n} a_{i} G_{P_{i}} .
$$

Nach unserem Satz 6 genügt es dann zu beweisen, dass für alle geschlossenen Kurven in $U^{*}$ das Integral $\int_{\gamma} F_{1}$ verschwindet. Aber gemäss unserem Satz 5 haben wir

$$
\begin{aligned}
\int_{\gamma} F_{1} & =\sum_{i} \int_{\gamma_{i}} F=\sum_{i} \int_{\gamma_{i}}\left(F-\sum_{j} a_{j} G_{P_{j}}\right) \\
& =\sum_{i} m_{i} 2 \pi a_{i}-\sum_{i} \sum_{j} m_{i} a_{i} \int_{\gamma_{i}} G_{P_{j}}=\sum_{i} m_{i} 2 \pi a_{i}-\sum_{i} m_{i} a_{i} 2 \pi=0 .
\end{aligned}
$$

Dies beweist unsere Behauptung.

Der Begriff der Umlaufzahl liefert in der Mathematik die Basis für die Behandlung der elementaren topologischen Eigenschaften von Kurven in der Ebene. Es war die Idee von Emil Artin (siehe [A]), auch die Homologie (in Dimension 1) mit Hilfe der Umlaufzahl zu definieren: Ein geschlossener Weg $\gamma$ in einem Gebiet $U$ heisst nullhomolog in $U$, wenn für alle Punkte $P$, die nicht in $U$ liegen, die Umlaufzahl $W(\gamma, P)$ Null ist. Die Voraussetzungen über den Weg $\gamma$ in unseren Sätzen 5 und 6 bedeuten also, dass $\gamma$ in $U$ nullhomolog ist. Für weiteren Aufschluss über die mit dem hier behandelten Thema zusammenhängenden elementaren Aspekte der Homologie und der Integration verweisen wir auf das klassische Buch von S. Lefschetz [Le], insbesondere auf den Abschnitt Integration and topology, p. 19-25. Dort werden auch einige weiterführende Entwicklungen angesprochen.

Acknowledgment: I am very grateful to U. Stammbach for the care he has given to the translation and the publication of my talk, and to Chr. Blatter for a critical reading of the text.

\section{Literatur}

[A] E. Artin: On the theory of complex functions. In The Collected Papers of Emil Artin, Addison Wesley, 1965; p. 513-522. Reprinted by Springer Verlag. Second edition, 1996.

[L1] S. Lang: Undergraduate Analysis, Springer Verlag 1983.

[L2] S. Lang: Complex Analysis, $3^{\text {rd }}$ edition, Springer Verlag 1993.

[Le] S. Lefschetz: Introduction to topology, Princeton University Press, 1949

Serge Lang

Yale University

New Haven

Connecticut 06520, USA 